\title{
Trends in mortality statistics in England and Wales with particular reference to AIDS from 1984 to April 1987
}

\author{
ANNA MCCORMICK
}

\begin{abstract}
Death certification should be able to provide accurate data on the number of deaths due to AIDS as a basis for predicting future deaths from the syndrome. Trends in deaths from other causes may identify conditions that have not been recognised to be associated with HIV infection. Mortality statistics with reference to AIDS in England and Wales were completed from death certificates. Increases in deaths from selected causes likely to be associated with AIDS or HIV infection suggested that in some patients with HIV infection, AIDS was not stated on the death certificate or subsequently notified by the doctor who signed the certificate. From calculations of excess deaths between the beginning of 1985 and the end of April 1987, compared with 1984 at least $\mathbf{4 9 5}$ deaths possibly associated with HIV infection were estimated to have occurred among men aged 15-54 during that period. In 261 AIDS or HIV infection was stated on the original or amended death entry as the cause of death, and of these 198 were included in the estimated number of excess deaths.

Accurate notification of the underlying cause of death and associated diseases is required for the precise monitoring of trends in mortality from AIDS and possible identification of unrecognised conditions associated with HIV infection.
\end{abstract}

\section{Introduction}

The medical practitioner who attends a person during his or her last illness ${ }^{1}$ or the coroner who holds a necropsy or inquest ${ }^{23}$ is required to state the cause of death on the prescribed form. The medical certificate of cause of death is laid out so that several conditions may be listed in sequence, the first being termed the underlying cause of

\footnotetext{
Medical Statistics Division, Office of Population Censuses and Surveys, London WC2B 6JP

ANNA MCCORMICK, MB, FFCM, senior medical statistician and consultant epidemiologist, PHLS Communicable Disease Surveillance Centre
}

death. Information from the certificate, supplemented with details about the deceased from the informant, who is usually a relative or friend, is entered on to the register of deaths, and a copy of the entry is sent by the local registrar to the Office of Population Censuses and Surveys (OPCS) for statistical analysis. The entry is a public document, a certified copy of which can be obtained by anyone who can provide sufficient information to identify the entry required. Doctors are requested to initial box $B$ on the reverse of the certificate if they expect to be later able to provide additional information about the cause of death to permit more precise statistical classification. This information is treated confidentially by OPCS and used solely for statistical purposes; the original copy of the entry available to the public is not altered as a result. Publications referring to data originating from these entries do not contain information that could identify an individual. Every original or amended death entry in which AIDS or HIV infection is mentioned is coded as International Classification of Diseases 279.1 (deficiency of cell mediated immunity) unless an underlying cause not usually associated with AIDS is stated.

The death certificate is an important legal and social document, but its use for epidemiological research is limited by the accuracy of the stated cause of death. ${ }^{4}$ Discrepancies are evident between both clinical diagnosis and diagnosis at necropsy and the underlying cause stated on the certificate, particularly for deaths reported to the coroner. $^{5}$

In New York city an increase of $34 \%$ in total mortality between 1980 and 1984 among men aged 15-64 who had never been married and who lived in areas with large homosexual populations was explained by the increase in deaths coded as AIDS. ${ }^{6}$ While total numbers of deaths due to drugs remained constant in New York city between 1981 and 1985 those due to pneumonia, endocarditis, and tuberculosis increased among intravenous drug abusers. No similar trends were noted in other high risk groups for AIDS or in the general population. It was suggested that this might have been the result of an unrecognised range of diseases related to HIV (R L Stoneburner et al, second international conference on AIDS. Paris, 1986).

The objectives of my study were to estimate trends in mortality related to HIV and the proportion of such deaths attributed to AIDS or HIV infection on the death certificate or in the supplementary information sent subsequently. 


\section{Methods}

The number of deaths due to all causes and selected causes and the population estimates were obtained from published and unpublished data at this office. Analysis was restricted to the $15-54$ age group because $91 \%$ of deaths among patients with AIDS reported to the Public Health Laboratory Service Communicable Disease Surveillance Centre fell within this age group, and deaths from other causes increased sharply above 54 years of age. Standardised mortality ratios were calculated by five year age groups for different age ranges by sex and by marital state. Marital state was not recorded on the register of deaths during 1981 .

I selected diagnoses that were likely to be stated as the underlying cause of death in patients dying from AIDS. The selection was based, firstly, on conditions also mentioned on the death certificates which stated AIDS or HIV infection to be the cause of death, and, secondly, on conditions mentioned on the certificates for which a diagnosis of AIDS or HIV infection was subsequently provided. I also made a search at OPCS for all entries for patients with AIDS reported to the Communicable Disease Surveillance Centre who were stated by the reporting clinician to have died but whose entry did not mention AIDS, HIV infection, or Kaposi's sarcoma; the conditions mentioned in these entries were included. Other conditions stated in the case definition of the WHO as being indicative of AIDS ${ }^{7}$ were also included. Causes of death occurring in fairly large but steadily decreasing numbers in this age group, such as carcinoma of the lung, were excluded to minimise the effect of trends due to causes other than HIV infection.

Excess deaths from the selected diagnoses together and separately were calculated by subtracting the number of deaths observed in 1984 from the number observed in each subsequent year until the end of April 1987, after adjusting for population changes such that excess deaths $(i)=$ observed deaths (i)-observed deaths ( 0$) \times($ population (i)/population ( 0$)$ ); where $(0)=1984$ and $(i)=1985,1986$, and 1987

Although some deaths due to AIDS occurred before 1984, this year was used as a baseline because of a change in policy in defining the underlying cause of death at the beginning of that year, making comparisons with data on some diagnoses from previous years difficult to interpret. Before 1984, when a terminal condition such as pneumonia was the only cause of death stated in part I of the certificate, and a major disease was cited in part II as a contributory factor, the terminal condition was usually coded as the underlying cause of death. Since 1984, depending on the terminal condition, the major disease mentioned in part II has more commonly been coded as the underlying cause. ${ }^{8}$ This change in the interpretation of rule 3 of the coding instructions of the WHO resulted in the transfer of the coding of about $11 \%$ of deaths that in 1983 would have been coded to a cause related to HIV to some other cause.

\section{Results}

Of 566881 deaths registered in 1984,26870 were among men aged 15-54 and 2455 among men aged 25-44 who had never been married. The proportions of men in the population aged 15-54 and 25-44 who had never been married increased during $1980-6$ from $37 \%$ to $40 \%$ and from $18 \%$ to $23 \%$, respectively.

For people aged 15-54 mortality ratios for all causes of death, standardised for age using 1980 as a baseline, fell steadily to 86.9 for men and 86.1 for women in 1986. The standardised mortality ratio for single men aged $25-44$, however, remained almost unchanged between 1984 and 1985 and increased in 1986 to $89 \cdot 7$ (fig 1). Mortality (for all causes of death) increased in single men aged 35-39 and 40-44 between 1984 and 1986 and in those aged 25-29 and 30-34 between 1985 and 1986, although the change was small (table 1).

In the 426 patients of all ages with AIDS reported to the Communicable Disease Surveillance Centre up to the end of April 1987 who were known by the centre to have died AIDS or HIV infection or Kaposi's sarcoma was

TABLE I-Mortality for all causes of death per 100000 population for single men aged 15-54 in England and Wales 1980-6

\begin{tabular}{lrrrrrr}
\hline \multirow{7}{*}{$\begin{array}{c}\text { Age group } \\
\text { (years) }\end{array}$} & 1980 & 1982 & 1983 & 1984 & 1985 & 1986 \\
\cline { 2 - 7 } & & 77 & 73 & 71 & 66 & 70 \\
\hline $15-19$ & 83 & 92 & 87 & 87 & 86 & 83 \\
$20-24$ & 98 & 117 & 118 & 112 & 102 & 108 \\
$25-29$ & 125 & 180 & 176 & 163 & 162 & 168 \\
$30-34$ & 179 & 245 & 273 & 250 & 272 & 273 \\
$35-39$ & 294 & 435 & 415 & 396 & 412 & 413 \\
$40-44$ & 469 & 722 & 727 & 696 & 729 & 691 \\
$45-49$ & 788 & 1184 & 1130 & 1123 & 1190 & 1083 \\
$50-54$ & 1257 & 114 & & & & \\
\hline
\end{tabular}

mentioned on the death certificate in 201, and AIDS or HIV infection was stated in supplementary information in a further 56. Sufficient data were available from the centre to identify the death entries of 101 of the remaining 169 patients. Table II shows the underlying cause of death stated on these entries.

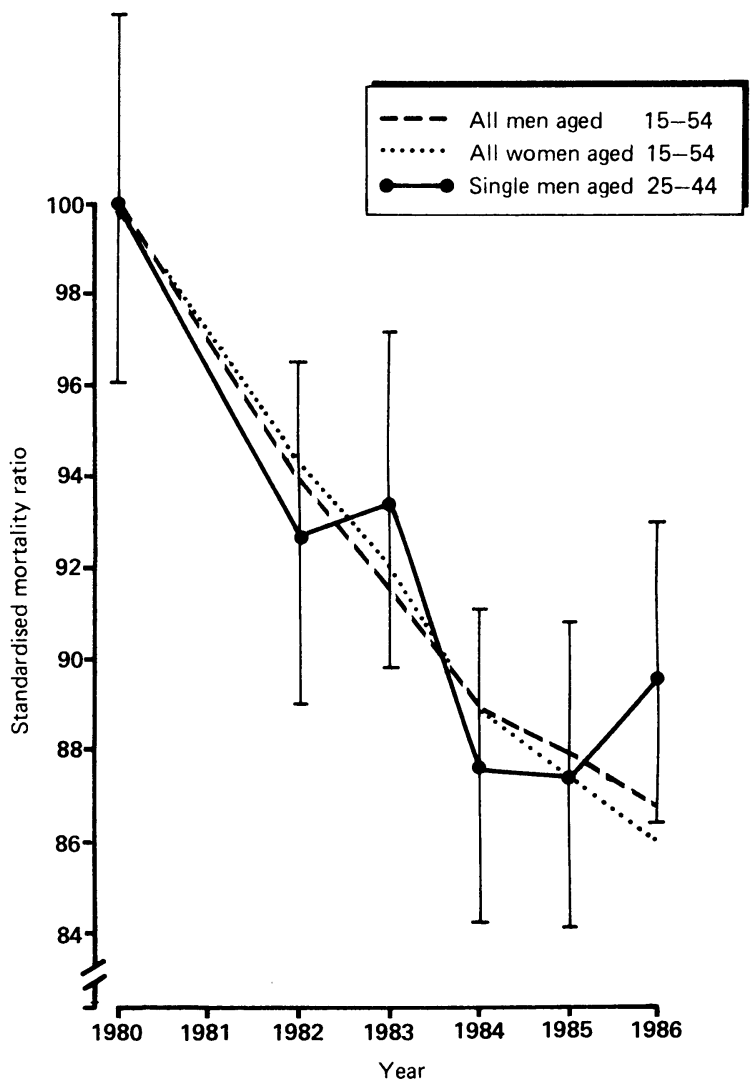

FIG 1-Standardised mortality ratios $(1980=100)$ with $95 \%$ confidence intervals in England and Wales 1980-6.

TABLE II-Underlying cause of death stated on death entries in cases of AIDS reported without mention of AIDS, HIV infection or Kaposi's sarcoma in England and Wales, 1980-April 1987

\begin{tabular}{|c|c|c|c|}
\hline Underlying cause of death & $\begin{array}{r}\text { No of } \\
\text { cases }\end{array}$ & Underlying cause of death & $\begin{array}{l}\text { No of } \\
\text { cases }\end{array}$ \\
\hline $\begin{array}{l}\text { Pneumonia or bronchopneumonia or } \\
\text { pneumonitis }\end{array}$ & 31 & $\begin{array}{l}\text { Mycobacterium avium intracellulare } \\
\text { infection }\end{array}$ & \\
\hline Chronic obstructive airways disease & 1 & Tuberculosis & \\
\hline Carcinomatosis & 3 & Cryptococcal meningitis & \\
\hline Carcinoma of mouth or tongue & 4 & Cerebral or cerebellar abscess & \\
\hline Sarcoma & 3 & Encephalitis & \\
\hline Carcinoma of lung & 2 & Pancreatitis & \\
\hline Brain tumour & 1 & Salmonella septicaemia & \\
\hline Lymphoma & 3 & Gastroenteritis & \\
\hline $\begin{array}{l}\text { Ischaemic heart disease or myocardial } \\
\text { infarction }\end{array}$ & 2 & Severe brain swelling & \\
\hline Toxoplasmosis & 2 & Presenile dementia & 1 \\
\hline Pneumocystis carinii pneumonia & 16 & Subarachnoid haemorrhage & \\
\hline Cytomegalovirus infection & 7 & Acute renal failure & \\
\hline Histoplasmosis & 1 & Open verdict & i \\
\hline
\end{tabular}

I compiled a list of 95 conditions likely to be stated as the underlying causes of death for patients with HIV infection. This included opportunistic and other infections, relevant cancers, immune deficiency, some mental illnesses, meningitis or encephalitis and intracranial abscesses, cerebrovascular accidents, pneumonias, and poisonings, as well as less specific conditions, such as cardiac, hepatic, and renal failure. Figure 2 shows standardised mortality ratios for these causes and for all other causes for single men aged 25-44; these have not been adjusted for the change in coding policy since 1984

Table III shows the deaths in men aged 15-54 for which one of the 95 
selected conditions was stated as the underlying cause, with the excess deaths compared with the number of deaths occurring in 1984. Adjustments for different changes in the populations of men of all marital states and those who had never married accounted for the higher number of excess deaths among men who had never married than for all men in 1985. Of these excess deaths, $90 \%$ were in men who had never been married. AIDS or HIV infection was stated as the underlying cause for 261 deaths. With the same method as for estimating total excess deaths there were 198 excess deaths for which AIDS or HIV infection was stated as the underlying cause. Figure 3 shows examples of some causes of death that increased in number. There

TABLE III-Deaths in men aged 15-54 from causes associated with AIDS or HIV infection in England and Wales 1984 to April 1987

\begin{tabular}{lccccc}
\hline & \multicolumn{2}{c}{ All marital states } & & \multicolumn{2}{c}{ Never married } \\
\cline { 2 - 3 } \cline { 6 - 6 } & Observed & Excess & & Observed & Excess \\
\hline 1984 & 5345 & & & 1790 & \\
1985 & 5522 & 134 & & 1988 & 159 \\
1986 & 5725 & 299 & & 2112 & 243 \\
1987 (to 30 April) & 1878 & 62 & & 671 & 42 \\
\hline Total & 13125 & 495 & & 4771 & 444 \\
\hline
\end{tabular}

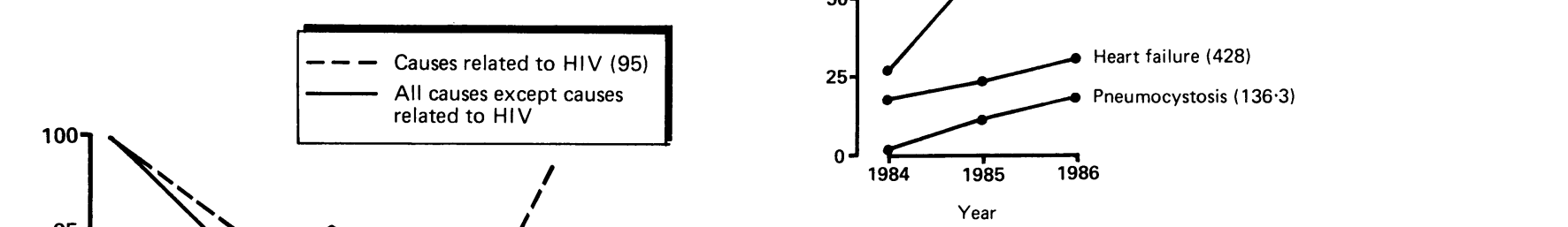

FIG 3-Deaths from selected causes (International Classification of Diseases code) in men aged 15-54 in England and Wales 1984-6.

between 1984 or 1985 and 1986 . The temporary increase in the standardised mortality ratio (all causes) in 1983 was confined to causes other than the 95 selected as possibly associated with HIV infection, suggesting that a factor other than HIV infection was responsible. The increase in the standardised mortality ratio (all causes) between 1985 and 1986, on the other hand, was almost exclusively accounted for by an increase in the standardised mortality ratio for the selected causes relating to HIV, suggesting that this trend was associated with infection with the virus.

Our method assumes that the impact of HIV infection can be measured by the upward trend since 1984 . However, standardised mortality ratios for all causes were falling among men aged 15-54 before the epidemic began and would have been expected to continue to fall. This fall was chiefly the result of decreasing numbers of deaths from causes common in this age group, such as neoplasms, particularly of the bronchus and lung, ischaemic heart disease, and cerebrovascular accidents, despite a gradual increase in the size of the population. If this trend has continued since 1984 among men not infected with HIV the number of excess deaths associated with infection has been underestimated. The change at the beginning of 1984 in the method of identifying the underlying cause of death among those conditions listed on the death certificate resulted in an $11 \%$ shift of coded deaths for all persons aged up to 75 from the 95 selected causes to other conditions. If such a shift were true for single men aged 25-44 the resulting adjustment for observed deaths due to the causes related to HIV in 1984 would suggest a standardised mortality ratio of 79 instead of 71 . Because of a dissimilarity in age and sex groups, however, extrapolation of the underlying trend beyond 1983 was thought to be unsuitable.

In $22 \%$ of deaths for which AIDS or HIV infection was finally coded as the underlying cause of death, this decision was reached only after the doctor signing the death certificate had supplied further information. The underlying causes of death other than AIDS stated on the certificates of known AIDS patients reported to the Communicable Disease Surveillance Centre were distributed among 27 diagnoses. Most were stated as pneumonia or bronchomortality for each five year age group of single men aged 25-44
The interrupted downward trend in the standardised mortality deaths each year, suggests that the pattern of mortality may have ratio for single men aged 25-44, although based on only about 2500

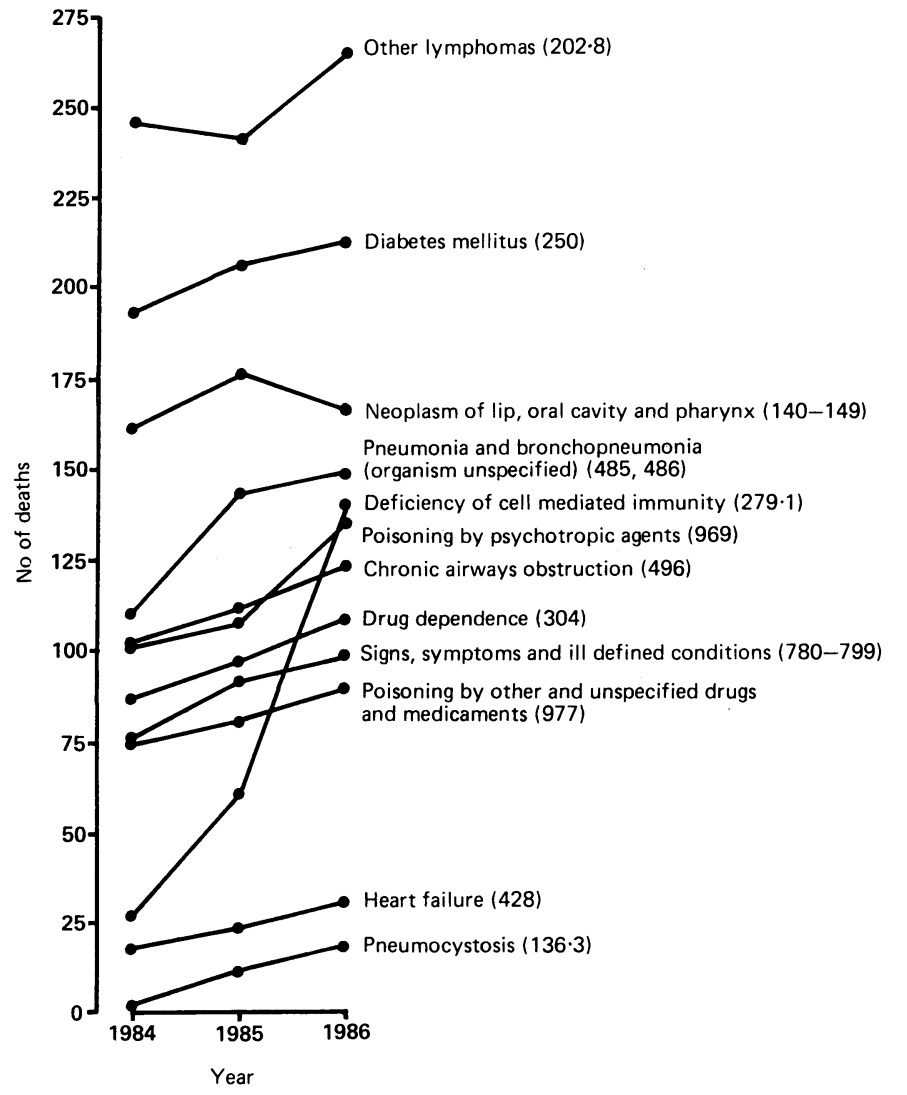

15-54 all deficit of 35 in the deaths from these 95 causes in women with HIV in men aged 15-54 showed a deficit between 1985 and the end of April 1987 for all men (2153) and for men who had never been married (745).

\section{Discussion}


pneumonia, organism unspecified or Pneumocystis carinii pneumonia; most of the remainder were conditions known to be associated with but not specific to AIDS.

As the excess deaths were calculated only from the beginning of 1985, although some deaths due to AIDS are known to have occurred before then, the number underestimates the total deaths due to AIDS since the syndrome was first recognised in the United Kingdom. The 1984 baseline was adjusted to take account of the small increase in the population estimates of men in each succeeding year.

Some of the excess deaths may not have been associated with HIV infection. If this were so the increase in deaths is difficult to explain, particularly as about $90 \%$ of them occurred in men who had never been married, and these men represent only $40 \%$ of men aged $15-54$. The deficit of deaths due to causes other than those possibly related to HIV infection suggests that the selection was generally appropriate. The deficit of deaths among women aged 15-54 emphasises the difference in the change in mortality between the sexes.

Infection with HIV may predispose to the development of clinical conditions that are not currently recognised as being associated with the virus. As the number of deaths after HIV infection increases analysis of other conditions mentioned on death certificates stating AIDS or HIV infection as the underlying cause of death and of trends in deaths attributed to other causes may help to identify a wider range of clinical manifestations.

Of the total 495 estimated excess deaths in men, in only 198 was the cause of death coded as AIDS by this office, leaving an estimated 297 coded as other causes. AIDS or HIV infection is therefore considerably understated as the cause of death. This is confirmed by the number of cases of AIDS reported to the Communicable Disease Surveillance Centre in which AIDS was not recorded on the death certificate. In some cases the correct diagnosis may not have been made at the time of death or the doctor may not have wished to mention AIDS on the certificate and did not subsequently send this information to this office. The increase in mortality rate in men who had never been married may be due to some manifestation of HIV infection other than the syndrome recognised as AIDS. Accurate reporting of the underlying cause and other conditions present at the time of death is necessary if illness not currently known to be associated with HIV infection is to be identified.

I am indebted to my statistician colleagues at this office, Michael Day and Tim Devis, for their invaluable help in preparing this paper.

\section{References}

Births and Deaths Registration Act 1953. London: HMSO, 1953:S22.

Coroners (Amendment) Act 1926. London: HMSO, 1926:S20.

3 Births and Deaths Registration Act 1953. London: HMSO, 1953:S23.

4 Alderson MR, Bayliss RIS, Clarke CA, Whitfield AGW. Death certification. Br Med $\mathcal{f}$ 1983;287:444-5.

5 Medical Services Study Group of the Royal College of Physicians of London. Death certification and epidemiological research. Br Med $\mathcal{F}$ 1978;ii: 1063-5.

6 Kristal AR. The impact of the acquired immunodeficiency syndrome on patterns of premature death in New York City. JAMA 1986;255:2306-10.

7 Centers for Disease Control. Revision of the CDC case definition for acquired immunodeficiency syndrome. $M M W R$ 1987;36(suppl):IS.

8 Office of Population Censuses and Surveys. 1984 Mortality Statistics. Series DH2. No 11. London: HMSO, 1985:v-vii.

(Accepted 17 March 1988)

\title{
Graves' disease: immunological and immunogenetic indicators of relapse
}

\author{
T W A DE BRUIN, J H BOLK, J K BUSSEMAKER, T STIJNEN, G M T SCHREUDER, \\ R R P DE VRIES, D VAN DER HEIDE
}

\begin{abstract}
The use of measurements of antibody to the thyroid stimulating hormone receptor and HLA-DR3 phenotype for predicting relapse of hyperthyroidism in patients with Graves' disease receiving medical treatment is controversial. Fifty eight new patients with Graves' disease were followed up prospectively for up to 96 months after treatment with antithyroid drugs for 12 months. The presence of antibody to the thyroid stimulating hormone receptor before the start of treatment, measured as immunoglobulins inhibiting binding of thyroid stimulating
\end{abstract}

\footnotetext{
Departments of Endocrinology, Internal Medicine, Medical Statistics, and Immunohaematology, University Hospital, 2333 AA Leiden, The Netherlands

T W A DE BRUIN, MD

J H BOLK, MD

J K BUSSEMAKER

T STIJNEN, PHD

G M T SCHREUDER, PHD

R R P DE VRIES, MD

D VAN DER HEIDE, PHD

Correspondence to: Dr T W A de Bruin, Department of Endocrinology, University Hospital, 3511 GV Utrecht, The Netherlands.
}

hormone, was not associated with relapse. Patients who remained positive for antibodies after treatment tended to relapse within six months, but no relation with long term relapse was found. HLA-Cw7 but not HLA-DR3 was significantly associated with relapse. The presence of HLA-DR4 was significantly associated with remission and with absence of antibodies to thyroid stimulating hormone receptor. HLA-DR4 may therefore protect against relapse of thyrotoxicosis by immunomodulation triggered by antithyroid drugs, which results in the synthesis of antibodies to the thyroid stimulating hormone receptor being inhibited.

\section{Introduction}

Graves' disease is characterised by hyperfunctioning of the thyroid, which is caused by antibodies to the thyroid stimulating hormone receptor. ${ }^{2}$ The natural course of the disease includes periods of spontaneous remission and recurrence. ${ }^{3}$ After treatment with thionamide drugs about $40-60 \%$ of patients relapse..$^{3-5}$ The ability to predict recurrence is important as treatment by operation or with iodine-131 may be better for such patients. As relapse of hyperthyroidism is probably mediated immunologically ${ }^{12}$ there may be immunological factors that are of prognostic value. Graves' disease is associated with the histocompatibility antigen HLA-DR3. ${ }^{26}$ Initially, the presence of HLA-DR3 and of antibody to the thyroid 